\title{
Abstract processing of syllabic structures in early infancy
}

Chiara Santolin ${ }^{1}$, Juan Manuel Toro ${ }^{1,2} \&$ Nuria Sebastian-Galles ${ }^{1}$

1. Center for Brain and Cognition, Universitat Pompeu Fabra

2. Catalan Institution for Research and Advanced Studies (ICREA)

Preprint submitted for peer-review on 27 September 2021

Author note:

Correspondence concerning this article should be addressed to Chiara Santolin; chiara.santolin@upf.edu

Edifici Merce Rodereda, Calle Ramón Trias Fargas, 25, 08018 Barcelona (Spain) 


\section{Abstract}

Syllables are the building blocks of early language acquisition. From birth onwards, infants preferentially segment, process and represent the speech into syllabic units, raising the question of what type of computations infants are able to perform on these perceptual units. Syllables are abstract phonological units structured in a way that allows to group speech sounds into sequences. The goal of the present research was to investigate 4-to5-month-old infants' ability to track the abstract internal structure of syllables, at a target age when the language system is not yet specialized on the sounds and the phonotactics of native languages. After familiarization to lists of syllables implementing either CVC (consonant-vowel-consonant) or CCV (consonant-consonant-vowel) structures, infants were presented with new syllables implementing both structures at test. Results showed that infants preferred test syllables that matched the familiar syllabic structure, especially the group of infants familiarized with CVC. Crucially, syllabic structures at test were implemented by new combinations of phonemes, forcing infants to encode some abstract properties of the familiarization stimuli, i.e., the internal syllabic structure. This study provides the first evidence of abstract processing of syllables as whole speech units in the first months of life. These findings contribute to advance our understanding of the type of computations young infants can perform on syllabic units.

Keywords: syllable, linguistic structure, abstract processing, learning, infancy 


\section{Introduction}

A long tradition of research shows that syllables are the primary unit of speech perception in infancy. Neonates discriminate speech sequences (bi or tri-syllabic utterances) differing in the number of syllables rather than phonemes, and show no sensitivity to variations in the number of phonemes composing bi-syllabic utterances (Bijeljac-Babic et al., 1993). This evidence suggests that few-day-old newborns preferentially track syllables instead of phonemes when processing the speech input (see also Bertoncini et al. 1995). Other studies reveal that 2-month-old infants form speech representations that most likely match syllables rather than phonetic segments (Jusczyk \& Derrah, 1987; Bertoncini et al., 1988). These investigations show that infants familiarized with syllables sharing a common phoneme (e.g., a vowel, as in bi or mi) discriminate among test syllables having both a new vowel and a new consonant, suggesting that infants do not categorize individual phonemes (i.e., they do not encode the common vowel as shared property among syllables) but track the whole syllabic unit (see also Eimas, 1999 for results on 3-4-month-olds). These pioneering studies propose that early infant perception and representation of speech are better structured in terms of syllables, and that syllables constitute the perceptual units for further linguistic processing. Interestingly, these studies leave open the possibility that, at such a young age, infants may be able to perform more complex computations on syllables than those described so far. In the present study, we aim at advancing this line of research, exploring whether young infants can encode the internal structure of syllables.

According to formal linguistics, syllables are abstract phonological constituents with hierarchical structures, whose main function is to group speech segments into sequences (e.g., Hockett, 1955; Halle \& Vergnaud, 1980). The syllable structure typically comprises a nucleus, characterized by the most sonorous segment (usually a vowel), an onset and sometimes a coda. Onset and coda are located at the edges of the syllable, and contain less sonorous segments with respect to the nucleus, such as consonants (De Lacy, 2007). Sonority (i.e., the audibility of speech sounds) defines the ordering of the segments within syllables. Such ordering creates temporal variations in the signal that allow listeners to chunk sounds into syllables (e.g., Selkirk, 1984; Clements, 1990).

A handful of studies show that young infants can form quite detailed representations of the phonemes composing syllables, and the patterns involving such phonemes. Fourmonth-old infants track sound patterns defining consonant-vowel (CV) syllables (e.g., 
fricative consonants always followed nasal vowels), and recognize such patterns in new syllables (Cristia et al., 2011). Similarly, 3-month-olds do not only categorize syllables with a constant phoneme (e.g., bi, be, ba, etc.) but also associate such phonetic categories to distinct visual objects (Mersad \& Dehaene-Lambertz, 2016; Mersad et al., 2021). Little is known, however, about whether or not infants around this age are able to form abstract representations of the syllabic structure as a whole, regardless of its individual constituents (phonemes or groups of phonemes).

The present study was designed to ask whether 4-to-5-month-old infants can track the internal structure of syllables (which determines the ordering of its constituents) and recognize such structure instantiated in several different syllables. We investigated this issue by implementing a generalization task that required infants to detect syllables' abstract structure from lists of syllables. We selected a target age range that precedes the establishment of native phoneme categories (Kuhl, 1979; Werker \& Tees, 1984), and native-languages phonotactics (Jusczyk et al., 1993; Mattys \& Jusczyk, 2001). Infants were first familiarized with a list of either CVC or CCV syllables (e.g., bor as CVC, plu as $\mathrm{CCV}$ ), then tested on their ability to discriminate between new CVC and new CCV syllables (e.g., pal vs. tro). We predicted that, if infants track the structure of the syllables presented at familiarization, they should show a preference for novel syllables having that same structure at test. To do so, infants would not only need to detect the syllabic structure they hear at familiarization, but also form a representation abstract enough to distinguish familiar implementations of that same syllabic structure from unfamiliar syllabic structures.

\section{Materials \& Methods}

\subsection{Participants}

Forty-eight infants of 4.5- to- 5.5 months of age participated in the study. Infants were assigned to either the CVC group (24 infants, mean age: 148 days) or the CCV group (24 infants, mean age: 150 days). Nineteen infants were excluded from the final sample because of difficulty to code head-turns (6), fussiness/crying (5), test performance was more than 2 standard deviations from the mean (4), parental interference (2), data did not meet the inclusion criterion (set prior to data collection) of at least 8 trials with looking times greater than $2 \mathrm{sec}$ (1), and technical error (1). All infants were healthy and full term, with no history of hearing or vision problems. The study was conducted in accordance with 
ethical standards of the Declaration of Helsinki and the protocol was approved by the local ethical committee (Parc de la Salut Mar, Barcelona). Caregivers signed a consent form before participating in the experiment and a small gift was given to them at the end of the experiment.

\subsection{Stimuli}

Ten CVC syllables and ten CCV syllables were used for the familiarization lists. Syllables were recorded by a female speaker, and concatenated into six strings. Syllables appeared once within each string, and were presented in random order. Strings were concatenated into a 3-min familiarization list, in which each string was repeated twice. Each syllable appeared twelve times during familiarization. There was 1-sec silence break between syllables and consecutive repetitions of the same syllable were avoided. Syllables composing the CVC familiarization list were: bor, dor, fil, fur, gol, kal, ker, pul, tar, and those composing the CCV familiarization list were: bro, dro, fli, fru, glo, kla, kre, plu, tra. Four additional syllables were recorded for the test phase, two CVC test items (pal, tor) and two CCV test items (pla, tro). A test trial consisted of an individual syllable repeated twelve times maximum (see Section 2.3 for information about trial duration). There were twelve test trials, three for each of the four test items, presented in random order. Consecutive repetitions of the same trial were avoided. All infants heard the same test trials regardless of the familiarization condition. In half of the test trials, CVC test items were presented (whose structure was familiar for infants familiarized with CVC, and unfamiliar for infants familiarized with CCV); in the other half of the test trials, CCV test items were presented (whose structure was familiar for infants familiarized with CCV, and unfamiliar for infants familiarized with CVC). Strings and lists of syllables were created using Praat (version 6.0.20). Duration (0.458 sec) and intensity (65 dB) of individual syllables were normalized across stimuli.

\subsection{Procedure}

We used the Head-turn Preference Procedure. Infants were seated on a caregiver's lap in a soundproof booth equipped with three computer screens while the caregiver listened to music over headphones to avoid interferences with the infant's behavior. Screens were placed in front (at $125 \mathrm{~cm}$ distance) and on the two sides (at $100 \mathrm{~cm}$ distance each) of the infant. The familiarization phase had a fixed duration of $3 \mathrm{~min}$, during which infants were presented with either the CVC or the CCV list. During the first 2 min of familiarization, infants heard the stimuli and watched a video-clip displaying neutral images (i.e., slow- 
moving clouds) on the central screen. During the last minute, a colored pinwheel moved across the screens to give infants the opportunity to practice head-turns to each side (e.g., Bouchon et al., 2015; Schott et al., 2021). ${ }^{1}$ At the beginning of each test trial, the pinwheel was displayed on the central screen until the infant fixated on it. At that point, a trained experimenter (blind to the stimuli) terminated the central pinwheel and triggered the appearance of one of the side-pinwheels. As soon as the infant made a head-turn towards the side pinwheel, one of the test items was played until the infant looked away for more than $2 \mathrm{sec}$, or until $24 \mathrm{sec}$ had elapsed (maximum trial duration). Once a trial ended, the central pinwheel reappeared. Test items were delivered by loudspeakers placed closed to the side screens. The procedure was set up using WISP, a custom-designed MATLAB software (Olson, 2017), which was also used to code looking times (see, for example, Thiessen \& Saffran, 2007; Lew-Williams et al., 2011; Santolin \& Saffran, 2019 for similar procedures). Looking time data were analyzed using JASP (0.14.1; Love et al., 2019) and visualized using $R$ (1.4.1106; RStudio Team, 2019). Stimuli and data are available at https://osf.io/eqgb4/.

\section{Results}

We ran a 2x2 mixed ANOVA with condition (familiarization to CVC vs. CCV) as betweenparticipant factor, and test structure (CVC vs. CCV) as within-participant factor. The critical interaction condition by test structure reached statistical significance $(F(1,46)=8.812, p=$ $\left.0.005, \eta_{p}^{2}=0.161\right)$, suggesting that infants' looking times at test differed as a function of familiarization (see Figure 1). Main effects were not statistically significant (test structure: $F(1,46)=0.079, p=0.780, \eta_{p}{ }^{2}=0.002$; condition: $\left.F(1,46)=2.155, p=0.149, \eta_{p}{ }^{2}=0.045\right)$. Looking times within each condition were examined with paired-samples t-tests. Infants familiarized with CVC syllables looked significantly longer to CVC test items than to CCV test items $(t(23)=2.750, p=0.011$, Cohen's $d=0.561)$. Average looking times were $10.160 \mathrm{sec}$ for CVC test items and $8.671 \mathrm{sec}$ for CCV test items. In contrast, infants familiarized with CCV failed to show a significant familiarity preference $(\mathrm{t}(23)=1.666, \mathrm{p}=$ 0.109 , Cohen's $d=0.340$ ). Average looking times were $10.910 \mathrm{sec}$ for CCV test items and $9.678 \mathrm{sec}$ for CVC test items.

\footnotetext{
1 The subdivision of the 3 familiarization minutes was slightly different for 4 infants, who spent 45 seconds (rather than 1 minute) practicing head-turns. Their average number of head-turns (3.25) was almost identical to the other infants (3.29).
} 
We compared looking times for test items across conditions to check for a priori preferences for one of the two syllabic structures, using independent-samples t-tests. Results show that both groups of infants looked similarly longer to CVC test items ( $\mathrm{t}(46)=$ $0.669, p=0.507$, Cohen's $d=-0.193)$. In contrast, infants in the CCV condition looked significantly longer to CCV test items than infants in the CVC condition $(t(46)=2.847, p=$ 0.007 , Cohen's $d=0.822$ ).

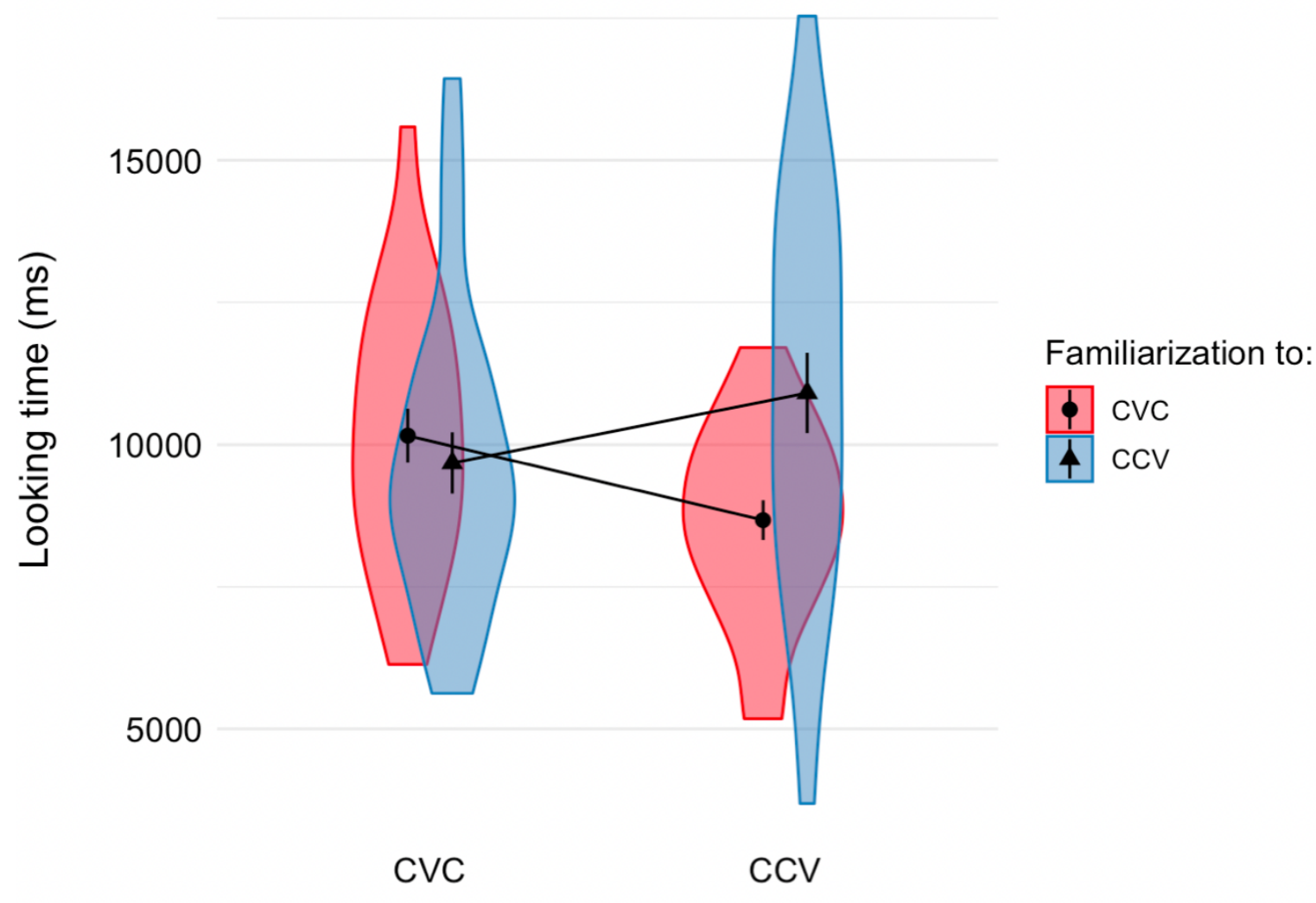

Test structure

Figure 1. Average looking times for test structures in each familiarization condition. Points represent observed group means, error bars represent standard errors, lines represent the critical interaction $(p=0.005)$.

\section{Discussion}

We investigated the ability of 4-to-5-month-old infants to track and generalize syllabic structures from lists of syllables. We found that infants looked longer to novel syllables that shared the same syllabic structure as those to which they were familiarized with than to novel syllables having a different syllabic structure. A significant interaction between familiarization (to either $\mathrm{CVC}$ or $\mathrm{CCV}$ ) and test structure (CVC and CCV) shows 
that infants preferred the structures they were familiarized with, even though test syllables were implemented by new constituents.

To the best of our knowledge, this research provides the first evidence of abstract processing of syllables in the first months of life. Processing phonetic information cannot account for infants' performance because test syllables are implemented by novel combinations of (familiar) phonemes. Indeed, to succeed in the task, infants must encode some abstract properties of the syllables, recognizing their internal structure regardless of the individual phonemes used to implement them. However, we observe a difference across syllabic structures. Contrary to infants in the CVC condition, the CCV group only shows a trend for the familiar structure. We suggest that this asymmetry may reflect a general preference for CVC over CCV structures, as CVC syllables are generally more frequent than CCV syllables across languages (e.g., Harris, 1983; Garcia-Jurado, 1985; Levelt, Schiller \& Levelt, 2000; Frota et al. 2005). It is thus plausible that the difference in frequency of syllabic structures that characterizes natural languages attenuate a preference for the familiar structure in the CCV condition.

Our study extends previous research demonstrating that infants process syllables very early in development, and spontaneously detect syllabic units from the speech input (e.g., Jusczyk et al., 1995; Eimas et al., 1999), which is interpreted as showing a holistic representation of the speech (see Halle \& Cristia, 2012 for a review). In the current study, we show that infants encode the abstract, internal structure shared among several syllable exemplars. Therefore, in addition to preferentially processing the speech into syllabic units, young infants seem also capable of performing some fine-grained computations on such units.

What type of information do infants use to track syllabic structures? It is possible that infants process the temporal variation in sonority that determines the position of phonemes (e.g., Räsänen et al., 2018). For instance, in CVC structures, there is a rise in sonority from onset (consonant) to nucleus (vowel), and a fall from nucleus to coda (another consonant). Infants may pick up on this pattern, and recognize it in the new set of test syllables. This possibility is also supported by neonatal research showing that 2-to-5day-old newborns are sensitive to such sonority variations in speech stimuli, being able to discriminate legal vs. illegal syllables based on sonority constraints (e.g., blif vs. Ibif; 
Gomez et al., 2014). Another possibility is that infants may detect changes in duration between vocalic and consonantal intervals. Such intervals indicate the onset and offset of vowels and consonants (and their clusters) composing a syllable (Ramus et al., 1999; see also Gasparini et al., 2021). For instance, CCV has a longer consonantal interval at the onset with respect to CVC. It is thus possible that infants track this pattern and use it to discriminate the new test syllables. Both these accounts would suggest that the information computed by infants during syllable processing is mainly acoustic in nature. Our results pave the way to further studies aimed at disentangling these possibilities. Additional experiments controlling for variations in sonority in the CVC syllables would allow us to explore whether infants would still show a preference for new CVC test syllables in the absence of clear acoustic changes. Another option would be increasing the sonority variations in the CCV syllables to see if this would trigger a preference pattern in the CCV group.

Previous studies show that infant sensitivity to syllables emerges with little language experience, and before important milestones of language acquisition are achieved (e.g., Bertoncini \& Mehler, 1981; Jusczyk \& Derrah, 1987; Bijeljac-Babic et al 1993). In parallel, research on other species (e.g., Toro \& Trobalon, 2005; Takahasi et al., 2010; Chen \& ten Cate, 2015) and computational modelling works (e.g., Frank et al., 2010; Doyle \& Levy, 2013) suggest that syllables can be used for the organization and segmentation of speech in the absence of language knowledge. These findings add up to the large body of work showing that some of the general mechanisms at the ontogeny of language acquisition are shared with other animals (e.g., Toro, Trobalon \& Sebastian-Galles, 2003; Saffran et al., 2008; Sonnweber et al., 2015; Santolin et al., 2016a; Santolin et al., 2016b; Toro, 2016; Rey et al., 2019, Petkov \& ten Cate, 2020). We interpret the results of the present research within a similar framework. If infants in our study are in fact using acoustic information (e.g., sonority variations, vocalic/consonantal intervals) to detect abstract syllabic structures, it is plausible that this early stage of syllable processing is a languagelearning step that does not necessarily require much language experience.

The present results contribute to advance our understanding of the type of computations infants can perform on syllabic units. This, in turn, could shed light on the nature of the privileged status of syllables as basic units of early speech perception. 


\section{Acknowledgments}

We give special thanks to Luca Bonatti for the helpful feedbacks on this work. We also thank Gonzalo Garcia-Castro, Konstantina Zacharaki, and Ege Ekin Özer for inspiring discussions on these results. We are grateful to Carlota Pagès for recording the stimuli, Pam Miller for proofreading the manuscript, and Xavier Mayoral, Cristina Dominguez, Silvia Blanch, Katia Pistrin of the Laboratori de Recerca en Infancia for their invaluable help during data collection. We are extremely grateful to the infants and the families who participated in the study. This research was supported by Juan de la Cierva Incorporación post-doctoral fellowship (IJC2019-041548-I / AEI / 10.13039/501100011033) awarded to CS, grants from Spanish Ministerio (PGC2018-101831-B-I00 AEI/FEDER EU) and Catalan Generalitat AGAUR (Clúster Emergent del Cervell Humà - CECH 001-P-001682, 2017 SGR 268) awarded to NSG, and grant from Spanish Ministerio (PGC2018-096641-B-I00) awarded to JMT. 


\section{References}

Bertoncini, J., \& Mehler, J. (1981). Syllables as units in infant speech perception. Infant Behavior and Development, 4, 247-260.

Bertoncini, J., Bijeljac-Babic, R., Jusczyk, P. W., Kennedy, L. J., \& Mehler, J. (1988). An investigation of young infants' perceptual representations of speech sounds. Journal of Experimental Psychology: General, 117(1), 21.

Bertoncini, J., Floccia, C., Nazzi, T., \& Mehler, J. (1995). Morae and syllables: Rhythmical basis of speech representations in neonates. Language and Speech, 38(4), 311-329.

Bijeljac-Babic, R., Bertoncini, J., \& Mehler, J. (1993). How do 4-day-old infants categorize multisyllabic utterances?. Developmental Psychology, 29(4), 711.

Bouchon, C., Floccia, C., Fux, T., Adda-Decker, M., \& Nazzi, T. (2015). Call me Alix, not Elix: Vowels are more important than consonants in own-name recognition at 5 months. Developmental Science, 18(4), 587-598.

Chen, J., \& Ten Cate, C. (2015). Zebra finches can use positional and transitional cues to distinguish vocal element strings. Behavioural Processes, 117, 29-34.

Clements, G. (1990). The role of the sonority cycle in core syllabification. Papers in Laboratory Phonology, 1, 283-333.

Cristia, A., Seidl, A., \& Gerken, L. (2011). Learning classes of sounds in infancy. University of Pennsylvania Working Papers in Linguistics, 17(1), Article 9. Retrieved from http://reposi- tory.upenn.edu/pwpl/vol17/iss11/19.

De Lacy, P. (2007). The interaction of tone, sonority, and prosodic structure. The Cambridge Handbook of Phonology, 281-307.

Doyle, G., \& Levy, R. (2013). Combining multiple information types in Bayesian word segmentation. Proceedings of the 2013 Conference of the North American Chapter of the Association for Computational Linguistics: Human Language Technologies, 117-126.

Eimas, P. D. (1999). Segmental and syllabic representations in the perception of speech by young infants. The Journal of the Acoustical Society of America, 105(3), 1901-1911.

Frank, M. C., Goldwater, S., Griffiths, T. L., \& Tenenbaum, J. B. (2010). Modeling human performance in statistical word segmentation. Cognition, 117(2), 107-125.

Frota, S., Freitas, M. J., Vigário, M., \& Martins, F. (2005). Prosodic and frequency effects on the development of syllable structure in European Portuguese. Paper presentation at 10th International Congress for the Study of Child Language, Berlin, Germany.

Garcia-Jurado, M. (1985). Los tipos silábicos del español. Revista Argentina de Lingüística, 1(2), 133-146. 
Gasparini, L., Langus, A., Tsuji, S., \& Boll-Avetisyan, N. (2021). Quantifying the role of rhythm in infants' language discrimination abilities: A meta-analysis. Cognition, Article 104757. https://doi.org/10.31219/osf.io/rmn5x

Gómez, D., Berent, I., Benavides-Varela, S., Bion, R. A., Cattarossi, L., Nespor, M., \& Mehler, J. (2014). Language universals at birth. Proceedings of the National Academy of Sciences, 111(16), 5837-5841.

Halle, M., \& Vergnaud, J. (1980). Three dimensional phonology. Journal of Linguistic Research, 1(1), 83-105.

Halle, P., \& Cristia, A. (2012). Global and detailed speech representations in early language acquisition. In S. Fuchs, M. Weirich, D. Pape, \& P. Perrier (Eds.), Speech planning and dynamics (pp. 11-38). Frankfurt am Main: Peter Lang.

Harris (1983). Syllable Structure and Stress in Spanish: A Nonlinear Analysis. Cambridge, MA: MIT Press.

Hockett, C. (1955). A manual of phonology. Chicago, University of Chicago Press.

Jusczyk, P. \& Derrah, C. (1987). Representation of speech sounds by young infants. Developmental Psychology, 23(5), 648.

Jusczyk, P. Friederici, A., Wessels, J., Svenkerud, V. \& Jusczyk, A. (1993). Infants' sensitivity to the sound patterns of native language words. Journal of Memory and Language, 32(3), 402-420.

Jusczyk, P. W., Kennedy, L. J., \& Jusczyk, A. M. (1995). Young infants' retention of information about syllables. Infant Behavior and Development, 18(1), 27-41.

Kuhl, P. K. (1979). Speech perception in early infancy: Perceptual constancy for spectrally dissimilar vowel categories. The Journal of the Acoustical Society of America, 66(6), 16681679 .

Love, J., Selker, R., Marsman, M., Jamil, T., Dropmann, D., Verhagen, J., ... \& Wagenmakers, E. J. (2019). JASP: Graphical statistical software for common statistical designs. Journal of Statistical Software, 88(1), 1-17.

Levelt, C., Schiller, N. \& Levelt, W. (2000). The acquisition of syllable types. Language Acquisition, 8(3), 237-264.

Lew-Williams, C., Pelucchi, B., \& Saffran, J. R. (2011). Isolated words enhance statistical language learning in infancy. Developmental Science, 14(6), 1323-1329.

Mersad, K., \& Dehaene-Lambertz, G. (2016). Electrophysiological evidence of phonetic normalization across coarticulation in infants. Developmental Science, 19(5), 710-722.

Mersad, K., Kabdebon, C., \& Dehaene-Lambertz, G. (2021). Explicit access to phonetic representations in 3-month-old infants. Cognition, Article: 104613. 
Olson, R. (2017). Wisconsin Infant Studies Program. Retrieved from https://bitbucket.org/rholson1/wisp/src/default/.

Petkov, C. \& Ten Cate, C. (2020). Structured sequence learning: Animal abilities, cognitive operations, and language evolution. Topics in Cognitive Science, 12(3), 828-842.

Ramus, F., Nespor, M., \& Mehler, J. (1999). Correlates of linguistic rhythm in the speech signal. Cognition, 73(3), 265-292.

Räsänen, O., Doyle, G., \& Frank, M. C. (2018). Pre-linguistic segmentation of speech into syllable-like units. Cognition, 171, 130-150.

Rey, A., Minier, L., Malassis, R., Bogaerts, L., \& Fagot, J. (2019). Regularity extraction across species: Associative learning mechanisms shared by human and non-human primates. Topics in Cognitive Science, 11(3), 573-586.

RStudio Team. (2019). RStudio: Integrated development environment for R RStudio, Inc. Retrieved from http://www.rstudio.com/.

Saffran, J., Hauser, M., Seibel, R., Kapfhamer, J., Tsao, F., \& Cushman, F. (2008). Grammatical pattern learning by human infants and cotton-top tamarin monkeys. Cognition, 107(2), 479-500.

Santolin, C., Rosa-Salva, O., Vallortigara, G., \& Regolin, L. (2016a). Unsupervised statistical learning in newly hatched chicks. Current Biology, 26(23), R1218-R1220.

Santolin, C., Rosa-Salva, O., Regolin, L., \& Vallortigara, G. (2016b). Generalization of visual regularities in newly hatched chicks (Gallus gallus). Animal Cognition, 19(5), 10071017.

Santolin, C., \& Saffran, J. R. (2019). Non-Linguistic Grammar Learning by 12-Month-Old Infants: Evidence for Constraints on Learning. Journal of Cognition and Development, 20(3), 433-441.

Schott, E., Mastroberardino, M., Fourakis, E., Lew-Williams, C., \& Byers-Heinlein, K. (2021). Fine-tuning language discrimination: Bilingual and monolingual infants' detection of language switching. Infancy. https://doi.org/10.1111/infa.12429

Selkirk, E. (1984). On the major class features and syllable theory. In M. Aronoff \& R. Oehrle (Eds.), Language sound structure (pp. 107-136). Cambridge Mass.: MIT Press.

Sonnweber, R., Ravignani, A., \& Fitch, W. T. (2015). Non-adjacent visual dependency learning in chimpanzees. Animal Cognition, 18(3), 733-745.

Toro, J. M., \& Trobalón, J. B. (2005). Statistical computations over a speech stream in a rodent. Perception \&Ppsychophysics, 67(5), 867-875.

Toro, J.M., Trobalón, J.B., Sebastián-Gallés, N. (2003). The use of prosodic cues in language discrimination tasks by rats. Animal Cognition, 6, 131-136. 
Toro, J. M. (2016). Something Old, Something New: Combining Mechanisms During Language Acquisition', Current Directions In Psychological Science, 25, 2, 130-134.

Takahasi, M., Yamada, H., \& Okanoya, K. (2010). Statistical and prosodic cues for song segmentation learning by Bengalese finches (Lonchura striata var.

domestica). Ethology, 116(6), 481-489.

Thiessen E.D., \& Saffran, J.R. (2007). Learning to learn: Infants' acquisition of stressbased strategies for word segmentation. Language Learning \& Development, 3, 73-100.

Werker, J. F., \& Tees, R. C. (1984). Cross-language speech perception: Evidence for perceptual reorganization during the first year of life. Infant Behavior and

Development, 7(1), 49-63. 